\title{
(2) OPEN ACCESS \\ Specialist palliative care services \\ response to ethnic minority groups with COVID-19: equal but inequitable —an observational study
}

\author{
Sabrina Bajwah (D) ,1,2 Jonathan Koffman (D) , ${ }^{1}$ Jamilla Hussain, ${ }^{3}$ \\ Andy Bradshaw (D) , ${ }^{3}$ Mevhibe B Hocaoglu, ${ }^{1}$ Lorna K Fraser (D) , 4 \\ Adejoke Oluyase (D) , ${ }^{1}$ Caitlin Allwin, ${ }^{1}$ Lesley Dunleavy, ${ }^{5}$ Nancy Preston, ${ }^{5}$ \\ Rachel Cripps, ${ }^{1}$ Matthew Maddocks, ${ }^{1}$ Katherine E Sleeman (1) , ${ }^{1,2}$ \\ Irene J Higginson (D) , ${ }^{1,2}$ Catherine Walshe (D) , Fliss E M Murtagh (D) , ${ }^{1,3}$ \\ on behalf of the CovPall study team
}

\begin{abstract}
${ }^{1}$ Cicely Saunders Institute of Palliative Care, Policy and Rehabilitation, King's College London, London, UK

${ }^{2}$ Department of Palliative Care, King's College Hospital NHS

Foundation Trust, London, UK ${ }^{3}$ Wolfson Palliative Care Research Centre, Hull York Medical School, University of Hull, Hull, UK

${ }^{4}$ Health Sciences, University of York, York, UK

${ }^{5}$ International Observatory on End of Life Care, Division of Health Research, Lancaster University, Lancaster, UK
\end{abstract}

\section{Correspondence to}

Dr Sabrina Bajwah, Cicely Saunders Institute of Palliative Care, Policy and Rehabilitation, King's College London, London WC2R 2LS, UK:

sabrina.bajwah@kcl.ac.uk

$\mathrm{IJH}, \mathrm{CW}$ and FEMM are joint senior authors.

Received 28 March 2021 Accepted 17 August 2021

\section{Check for updates}

(c) Author(s) (or their employer(s)) 2021. Re-use permitted under CC BY. Published by BMJ.

\begin{tabular}{l}
\hline To cite: Bajwah S, \\
Koffman J, Hussain J, \\
et al. BMJ Supportive \& \\
Palliative Care Epub ahead \\
of print: [please include Day \\
Month Year]. doi:10.1136/ \\
bmjspcare-2021-003083
\end{tabular}

\section{ABSTRACT}

Objectives To develop insights into response of palliative care services caring for people from ethnic minority groups during COVID-19.

Methods Cross-sectional online survey of UK palliative care services response to COVID-19. Quantitative data were summarised descriptively and $\chi^{2}$ tests used to explore relationships between categorical variables. Free text comments were analysed using reflexive thematic analysis.

Results 277 UK services responded. 168 included hospice teams (76\% of all UK hospice teams). Services supporting those from ethnic minority groups were more likely to include hospital $(p<0.001)$ and less likely to include hospice $(p<0.001)$ or home care teams ( $p=0.008) .34 \%(93 / 277)$ of services had cared for patients with COVID-19 or families from ethnic minority groups. 66\% (61/93) of these services stated no difference in how they supported or reached these groups during the pandemic.

Three themes demonstrated impact of policy introduced during the pandemic, including: disproportionate adverse impact of restricted visiting, compounded communication challenges and unmet religious and faith needs. One theme demonstrated mistrust of services by ethnic minority groups, and the final theme demonstrated a focus on equal and individualised care.

Conclusions Policies introduced during the COVID-19 pandemic may have adversely impacted those from ethnic minority groups making these at-risk populations even more vulnerable. The palliative care response may have been equal but inequitable. During the

\section{Key messages}

What was already known?

- COVID-19 has disproportionately affected ethnic minority groups.

What are the new findings?

- Policies may have disproportionately impacted ethnic minorities at end of life.

- Palliative care response may have been equal but inequitable.

What is their significance?

- Systemic steps, including equality impact assessments, are urgently needed to address inequity.

- Focus on individualised care may be insufficient to provide equitable care.

para-COVID-19 period, systemic steps, including equality impact assessments, are urgently needed.

\section{INTRODUCTION}

The disproportionate impact of COVID-19 (ie, increased likelihood of infection and death) on those from ethnic minorities is well documented. ${ }^{1}$ Suggested reasons include existing health inequalities, housing conditions (including multigenerational factors), public-facing occupations and structural racism. ${ }^{23}$

Prior to the pandemic, $\mathrm{UK}^{4-6}$ and international $^{7} 8$ evidence has demonstrated inequity in the delivery and provision of palliative care (eg, access to services, place of care/death, late referrals) for those from ethnic minority groups. Within 
the UK, the term 'ethnic minorities' is used to refer to all ethnic groups except the White British group. Ethnic minorities include White minorities, such as Gypsy, Roma and Irish Traveller groups. ${ }^{9}$ While there is limited evidence from a single UK centre suggesting that patients from ethnic minorities may have had later referral to palliative care during the pandemic, ${ }^{10}$ we do not know more widely how palliative care services responded to the needs of these patients and families. There is also an absence of evidence of challenges services experienced in caring for patients and their families from ethnic minority groups and how they have adapted their services to meet these challenges. By 2040, demand for palliative care is projected to significantly increase in the $\mathrm{UK},{ }^{11}$ and the prevalence of those from ethnic minority groups is expected to rise from $12.7 \%$ in 2011 to $30.3 \%$ in $2051 .^{12}$ It is important that palliative care services learn from the pandemic to shape practice and policy in equitable ways that meets the needs of all.

This study aims to map and develop insights into the response of specialist palliative care services caring for patients and families from ethnic minority groups during the first wave of the COVID-19 pandemic.

\section{METHODS}

\section{Design and participants}

CovPall is a multicentre observational study of palliative care during the COVID-19 pandemic. ${ }^{13}$ We report data from the UK nations (England, Wales, Scotland and Northern Ireland) only from an online, crosssectional survey of clinical leads of palliative care and hospice services. International data were excluded as there was limited knowledge of local context, constraining interpretation.

Services providing hospice and specialist palliative care across inpatient palliative care, hospital palliative care, home palliative care and home nursing settings were eligible for participation and were recruited through palliative care and hospice organisations (Sue Ryder, Hospice UK, Marie Curie, European Association of Palliative Care, Together for Short Lives, and the palliativedrugs.com and www.pos-pal. org network) between April and July 2020. Within these sites, service leads (medical or nurse directors/ clinicians) or their selected nominees were eligible to complete the survey. The CovPall protocol is registered (ISRCTN16561225) and these survey results are reporting according to Strengthening the Reporting of
Observational Studies in Epidemiology ${ }^{14}$ and Checklist for Reporting Results of Internet E-Surveys ${ }^{15}$ checklists.

\section{Survey and data collection}

This survey was developed through iterations within the CovPall team and piloted in expert and patient public involvement consultations. Research Electronic Data Capture ${ }^{16}$ was used to securely build and host the survey which aimed to understand how specialist palliative care and hospice services responded to the COVID-19 pandemic. The survey comprised 72 closed text and 94 free text responses and is reported in full in the main study paper. ${ }^{13}$

This paper focuses on UK services who answered survey questions about care of those from ethnic minority groups. For the quantitative data items, we analysed the following variables by whether or not they encountered patients or families with COVID-19 from ethnic minority groups: UK regions, setting (inpatient hospice palliative care team, hospital palliative care team, home palliative care team, home nursing team), management type (public, charitable, private/ other), type of patient (adult, children or both), presence of suspected or confirmed COVID-19, number of confirmed or suspected COVID-19 cases per service and disease severity. From the free text comments, we analysed one closed-ended and two open-ended questions (see table 1). The responses provided were responses made by service leads (or their nominees) on behalf of the service in which they worked.

\section{Data analysis}

Anonymised quantitative data items were summarised descriptively. Continuous variables were expressed as means (SD), or medians (IQR) if the assumption of normality was not met. Categorical variables were expressed as counts and percentages; $\chi^{2}$ tests were used to explore relationships between categorical variables with a $p$ value of 0.05 as the cut-off to determine significance level. SPSS (V.26) was used for statistical analysis.

The analysis of the free text data was informed by guidance specifically developed for postal or online questionnaires. ${ }^{1718}$ We used reflexive thematic analysis $^{19}$ as a method to guide analysis in which two researchers ( $\mathrm{JK}$ and $\mathrm{JH}$ ) independently familiarised themselves with the data for each comment box by reading all the responses. Data for each question

Table 1 Questions from CovPall survey focusing on patients and families from ethnic minority groups

\begin{tabular}{ll}
\hline Questions taken from section 4 of the survey, titled: 'How have your services changed in response to COVID-19?' \\
$\begin{array}{ll}\text { Closed ended } & \text { Question 4.20: Have you encountered patient or families with COVID-19 who are from black and minority ethnic groups? } \\
\text { Yes/No (if yes, free text box opened) }\end{array}$ \\
$\begin{array}{ll}\text { Open ended } & \text { Question 4.20a: Are there any differences in how you are supporting or reaching them? } \\
& \begin{array}{l}\text { Question 4.21: Are there any groups (different religions, cultures) where you have found supporting the individual needs of } \\
\text { people affected by COVID-19 particularly challenging? }\end{array}\end{array}$ \\
\hline
\end{tabular}


were initially analysed separately, before coming together. Data were analysed across all questions before the creation of themes. Notes were made of any potential codes from the data set by identifying recurring words or units of meaning. During the analysis, the researchers employed an abductive approach in which induction and deduction were combined. ${ }^{20}$ That is, coding and theme development were initially driven by the content of the comments in the data and required a continual bending back on oneself, questioning and querying the assumptions made in interpreting and coding the data. Central to this was reflective and critical engagement with the data and analytical process more generally. During this process, codes were amalgamated, or new codes were created as differences in meaning were identified. A third researcher (SB) reviewed the first and second-order coding decisions. Any discrepancies or disagreements were discussed between $\mathrm{SB}$, JK and JH and, where necessary, adjustments made to achieve consensus. These data were then subsequently discussed with the wider study group who have various backgrounds to prevent one-sided interpretation of the data. To preserve context, the anonymised comments have been presented in full. As per recent recommendations, we will be using the term 'ethnic minority groups' in our reporting rather than 'black and minority groups'. ${ }^{21}$ This term is used to represent a heterogenous rather than homogenous group. Where data are available, we have referred to the individual ethnic group.

\section{RESULTS}

\section{Descriptive data}

We received responses from 277 UK palliative care services between 23 April 2020 and 31 July 2020, of which 168 included hospice services (equating to $\sim 76 \%$ of hospice services in the $\left.\mathrm{UK}^{22}\right)$. Of responding organisations, $34 \%(\mathrm{n}=93)$ had cared for patients and families from ethnic minority groups (table 2). Twenty-three per cent $(n=35)$ included inpatient hospice palliative care teams, 49\% $(n=60)$ hospital palliative care teams, $30 \%(n=43)$ home palliative care teams and $31 \%(n=26)$ home nursing teams. Nearly two-thirds (59\%) of services offered care in more than one setting. The greatest number of services supporting those from ethnic minority groups were in London and East England $(\mathrm{n}=44)$, South East $(\mathrm{n}=11)$, North West $(n=10)$ and the Midlands $(n=10)$. Services who had supported those from ethnic minority groups were more likely to have hospital palliative care teams $\left(\chi^{2}=15.21, p<0.001\right)$ and less likely to have inpatient hospice $\left(\chi^{2}=30.11, \mathrm{p}<0.001\right)$ or home palliative care teams $\left(\chi^{2}=7.05, \mathrm{p}=0.008\right)$.

Characteristics of the survey sample and provision of care to those from ethnic minority groups are provided in table 2 .

\section{Free text data analysis}

We present five themes that demonstrate how services supported or reached patient or families with COVID-19 from minority ethnic groups.

\section{Theme 1: disproportionate impact of restricted visiting}

While everyone was required to endure not being able to see their loved ones at the end of life, respondents noted that during the pandemic, families of patients from ethnic minority groups were particularly affected by restrictions on visiting. Services reported that families struggled as they were unable to fulfil religious and culturally prescribed responsibilities. This involved providing face-to-face physical care and emotional support to their family member. Visiting restrictions had a disproportionate adverse impact in those ethnic minority groups that would traditionally have large numbers of family members involved in providing care and support and/or decision-making, and where it was important for the wider community to visit and support in times of illness. Many of these services struggled to meet the increased needs of these patients and families.

We had a lot of distress from Muslim families about the strict visitation policy, as high numbers of people at the time of death is associated with a better afterlife. (Service in North West England)

I think the visiting restrictions have hit Asian and travelling communities harder than other groups. (Service in East England)

Some patients of particular cultures/religions often involve large numbers of visitors to support them, which is more difficult now with the restrictions on visiting. (Service in Yorkshire and the Humber)

\section{Theme 2: compounded communication challenges}

Communication challenges represented another overriding issue. Services reported that this was particularly an issue for patients and families from ethnic minority groups where English was not their first language. While wearing of personal protective equipment made conversing difficult for all, the barriers to communication for these individuals were compounded-due to the visiting restrictions, neither family members nor professional interpreters could be physically present to interpret. To mitigate against this, staff tried to use telephone interpreters through personal protective equipment and some staff members used language translation software applications loaded onto their mobile phones. However, services reported that these potential solutions to communication challenges were limited in scope, availability and effectiveness.

There was an issue with language barriers among those who don't speak English and no longer have their family members to translate for them ... In addition, translators were not available in [the] Trust. (Service in London) 
Table 2 Comparison of the characteristics of responding UK palliative care services that encountered patients or families with COVID-19 from ethnic minority groups and those of services that did not

\begin{tabular}{|c|c|c|c|}
\hline & $\begin{array}{l}\text { UK services that supported } \\
\text { ethnic minority groups } \\
\mathrm{n}(\%)^{*}\end{array}$ & $\begin{array}{l}\text { UK services that did not support } \\
\text { ethnic minority groups } \\
\mathrm{n}(\%)^{*}\end{array}$ & $\begin{array}{l}\chi^{2} \text { analysis with } P \\
\text { value }\end{array}$ \\
\hline Total responses & $93(36.6)$ & $161(63.4)$ & \\
\hline \multicolumn{4}{|l|}{ UK regions } \\
\hline \multicolumn{4}{|l|}{ England } \\
\hline North East and Yorkshire & $8(23.5)$ & $26(76.5)$ & \\
\hline North West & $10(30.3)$ & $23(69.7)$ & \\
\hline Midlands & $10(41.7)$ & $14(58.3)$ & \\
\hline London and East England & $44(81.5)$ & $10(18.5)$ & \\
\hline South East and South West & $13(21.3)$ & $48(78.7)$ & \\
\hline Missing & $8(16.7)$ & $40(83.3)$ & \\
\hline Wales, Northern Ireland and Scotland & $8(17.4)$ & $38(82.6)$ & \\
\hline Missing & - & $4(100)$ & \\
\hline \multicolumn{4}{|l|}{ Setting $\dagger$} \\
\hline \multicolumn{4}{|l|}{ Inpatient hospice palliative care team } \\
\hline No & $58(56.9)$ & $44(43.1)$ & \\
\hline Yes & $35(23)$ & $117(77)$ & $\chi^{2}=30.11, p<0.001$ \\
\hline \multicolumn{4}{|l|}{ Hospital palliative care team } \\
\hline No & $33(25.2)$ & $98(74.8)$ & \\
\hline Yes & $60(48.8)$ & $63(51.2)$ & $\chi^{2}=15.21, p<0.001$ \\
\hline \multicolumn{4}{|l|}{ Home palliative care team } \\
\hline No & $50(45.9)$ & $59(54.1)$ & \\
\hline Yes & $43(29.7)$ & $102(70.3)$ & $\chi^{2}=7.05, p=0.008$ \\
\hline \multicolumn{4}{|l|}{ Home nursing team } \\
\hline No & $67(39.4)$ & $103(60.6)$ & \\
\hline Yes & $26(31)$ & $58(69)$ & $\chi^{2}=1.73, p=0.19$ \\
\hline \multicolumn{4}{|l|}{ Type of management } \\
\hline Charitable/non-profit & $33(24.3)$ & $103(75.7)$ & \\
\hline Public & $57(57.6)$ & $42(42.4)$ & \\
\hline Private/other & $2(13.3)$ & $13(86.7)$ & \\
\hline Missing & $1(25)$ & $3(75)$ & \\
\hline \multicolumn{4}{|l|}{ Patients } \\
\hline Adult only & $87(38.3)$ & $140(61.7)$ & \\
\hline Children only & $1(6.7)$ & $14(93.3)$ & \\
\hline Both & $3(30)$ & $7(70)$ & \\
\hline Missing & $2(100)$ & - & \\
\hline \multicolumn{4}{|l|}{ Approximate number of confirmed COVID-19 cases per service } \\
\hline Median (IQR) & $50(12.8,90)$ & $7.5(3,20)$ & \\
\hline Total & 88 & 124 & \\
\hline Missing & 5 & 37 & \\
\hline \multicolumn{4}{|l|}{ Approximate number of suspected COVID-19 cases per service } \\
\hline Median (IQR) & $15(10,30)$ & $6(3,18.5)$ & \\
\hline Total & 71 & 118 & \\
\hline Missing & 22 & 43 & \\
\hline \multicolumn{4}{|l|}{ Disease severity } \\
\hline $\begin{array}{l}\text { Patients who are severely ill or dying due mainly to COVID-19 but without } \\
\text { pre-existing illnesses or comorbidities. }\end{array}$ & $60(55.6 \%)$ & $48(44.4 \%)$ & \\
\hline $\begin{array}{l}\text { Patients with pre-existing illnesses/comorbidities as well as COVID-19 who } \\
\text { are severely ill or dying not previously known to palliative care. }\end{array}$ & 77 (41.4\%) & $109(58.6 \%)$ & \\
\hline Patients already known to service who now have COVID-19. & $58(46 \%)$ & $68(54 \%)$ & \\
\hline
\end{tabular}

${ }^{*} \mathrm{n}$ of value and corresponding percentages are presented, unless otherwise indicated.

tServices could tick more than one category if applicable.

Face to face interpreters largely not available (though there are remote service for difficult conversations this can feel less personal). (Service in East Midlands)
Telephone interpreting service was challenging and difficult through masks. (Service in Scotland) 
Theme 3: religious and faith needs at the end of life

Multiple inter-related issues were present concerning the place of religion and faith (rules, rites, regulations and practices) among patients with COVID-19 and their families from ethnic minority groups. With restricted visiting, responsibility was often devolved to faith leaders to provide support which had previously been provided by large groups of family members. However, face-to-face access to faith leaders-who, at times, were themselves at high risk-from ethnic minority groups was often restricted. This led to delays providing face-to-face support, or a default to remote support.

At the start of the outbreak/peak [we had a] challenge in accessing faith leaders from BAME [Black Asian and Ethnic Minority] community. (Service in East Midlands)

Yes, relatives belonging to small faith groups unable to access support. (Service in North West England)

Our $\operatorname{Iman}[\mathrm{m}]$ is asthmatic and so stopped visiting the hospital. It took a bit of time to find another who was willing to come in. (Service in London)

Care after death compounded religious and faithbased issues for many patients and their families from ethnic minority groups. Importantly, changes in policylimiting access to the body after death caused distress. For example, the Jewish ritual of 'Tahara' and Muslim rituals of 'Ghusl' and 'Kafan' that involved relatives preparing a body for burial by washing, reciting prayers and psalms and dressing them in a shroud were not permitted. In addition, services expressed difficulty in meeting the religious need for burial within 24 hours after death and the challenges this posed.

Difficulties with initial guidance around not allowing families to touch bodies (common in some cultures) so not able to perform usual post-death rituals (ritual washing of bodies). (Service in East England)

Reduced visiting and changes to care of the body after the death and funeral arrangements is impacting some families more than others. Some of these differences are likely to be influenced by religious beliefs and culture. (Service in London)

Supporting Muslim and Jewish families to have burial within 24 hrs of death has been challenging. (Service in London)

Positive examples were noted of reaching out to external faith groups to support patients to reduce the impact on ethnic minority groups. Some services explicitly stated they were well versed in serving a diverse population and already had established links with their local ethnic minority communities who they had effectively reached out to during the first wave of the pandemic.

We have a very religious and culturally diverse population, so we are really used to understanding their needs and having support available. We have the X Community of Mosques that we have a good relationship with. (Service in North West England)

We had Quran cubes brought in for dying patients. (Service in North West England)

Theme 4: mistrust

Mistrust was reported among respondents' perceptions in discussing advance care planning and ceilings of treatment. This was specific to certain communities, for example, the strictly orthodox Haredi Jewish community.

Certain cultures and religions can sometimes regard our service as being opposed to their beliefs (e.g., Orthodox Jewish community). This poses some challenges in providing care. (Service in London)

There were also suggestions of a general mistrust of healthcare services which may have been related to previous poor experiences in the healthcare system and concerns about rationing of services.

Patients let down by other services [and] difficult to engage. (Service in South East England)

Some suggestion that certain patients from some groups and their relatives are more concerned about rationing of services. (Service in East England)

Theme 5: equal service response with a focus on individualised care When service providers were asked if there were any differences in how they supported or reached ethnic minority groups, 66\% (61/93) of services that had looked after patients and families from ethnic minority groups indicated that they had not adopted a different approach and all patients had been treated equally. Some of these respondents suggested the needs of ethnic minority groups were no different and consequently did not consider changes were necessary.

No- the support they required was not different. (Service in London)

No different to other patients/families with COVID-19. (Service in South East England)

No difference at all. (Service in North East England)

Offering same support as others. (Service in London)

No - all patients/families/carers offered same support regardless of ethnic origin. (Service in North West England)

This view of equality seemed to be supported by the perception that assessment of patient needs was individualised and that any care subsequently delivered to them was therefore matched appropriately to needs.

Every patient seen by the SPC [Specialist Palliative Care] team has a holistic assessment to identify individual needs. (Service in London) 
We aim to support all patients as individuals with the offer of support determined by their needs. (Service in Yorkshire and the Humber)

A few services recognised that they had not changed the care provided to patients from ethnic minority groups in the pandemic, and emphasised it was important to examine their response to identify if changes would be required as the pandemic progressed. Other services reported that some communities had historically been difficult to engage and during the pandemic this situation had become amplified, with a perception that certain groups were more likely to be resistant to care offered by services.

\begin{abstract}
We are not aware of any change in our practice or service delivery, but [we] are currently reporting on our response with particular reference to BAME communities. (Service in London)

Some of the areas we work in have always been difficult to really access, some families now closing the doors to us, still trying the same way, haven't changed approach. (Service in West Midlands)
\end{abstract}

Some providers stated they had adapted and adopted creative approaches to address the needs of those from ethnic minority groups. These strategies were built on pre-existing relationships and links with community groups. Recognising the importance of actively reaching out to these groups, some offered outreach care or sent emails or telephoned families to inform them care was still available despite the pandemic.

A lot of people [from ethnic minorities] are so frightened that they are not allowing the help and support they need until it is a crisis. (Service in East England)

[we are] conscious of their concerns/fears with respect to prognosis from COVID. (Service in London)

We sent ...emails and telephoned to say the service is here to support them. (Service in West Midlands)

Prior to COVID we had been working to increase our diversity but were finding it difficult to engage with different cultures i.e. our local Asian communities due to the way that they care for their elders within their own families, language barriers have also been an issue in trying to communicate what services we could provide, however this work that we intend to continue, unfortunately the social distancing issues from C19 have put a stop on the projects and meetings that we had commenced. (Service in Yorkshire and the Humber)

Increased awareness of impact of COVID on BAME communities and acknowledgement by Trust with support sessions established. (Service in London)

For other service providers, there appeared to be little consideration about issues that did not go well. For example, if the challenges associated with poor communication were considered, the responsibility to resolve this was placed with the patient and their family. Or, when services were asked about specific challenges related to different religions and cultures, this 'deficit model' ${ }^{23}$ implied 'lack of understanding' or 'struggle' as a deficit or lack within the ethnic minority group, rather than something to be considered, understood and addressed by the services.

[they have] different expectations. (Service in London)

[they are] misunderstanding about the role of pall care. (Service in East England)

However, some services recognised the importance of self-reflection and adapting services to meet the needs of these patients and families.

End of life situations are dealt with differently with all families - some people will always see things differently - it is our role to adapt to provide the support that they specifically require and will accept. (Service in North East England)

\section{DISCUSSION}

Specialist palliative care services have faced challenges in meeting the needs of those from ethnic minority groups during the COVID-19 pandemic. This study aimed to map and develop insights into the response of specialist palliative care services caring for patients and families from ethnic minority groups during the first wave of the COVID-19 pandemic.

Our findings suggest that while service providers may have perceived they were treating all patients and families equally through individualised care, policies around visiting and after death care may have impacted ethnic minority groups more. This may have resulted in equal but inequitable care at the end of life for these groups. Equality means every individual or group of people is given the same resources or opportunities to achieve the same outcome. ${ }^{24}$ In contrast, inequity relates to unfairness or injustice; reflecting a sense that different resources might be needed to achieve similar outcomes. ${ }^{24}$ In delivering equitable healthcare, some patients and families from ethnic minority groups may require more resources. While we may have a perception that by treating everyone the same/equally we are doing good and being fair, this is not always the case. Treating all patients and families equally regardless of ethnicity is likely to lead to unequal outcomes and is therefore likely to be inequitable care. Another strong theme in our findings was that services had focused on delivering individualised care. While the individualised approach adopted in palliative care is important, focusing solely on individualised care may overlook systemic and organisational changes needed to ensure equitable delivery of care for those from ethnic minority groups. 
Health inequality models that include the WHO Commission on Social Determinants of Health ${ }^{25}$ help us to understand that health inequalities exist at multiple complex interacting levels and are the result of many factors, including socioeconomic position, psychosocial and health system factors. ${ }^{25}$ Inequalities in health, healthcare access and quality of care are ingrained in healthcare systems. Many members of ethnic minority groups have lower socioeconomic status, have two or more coexisting conditions and reside in conditions that impede social distancing. A recent systematic review conducted prior to the pandemic highlighted persistent inequalities in hospice care provision for a number of groups, including those from ethnic minorities. ${ }^{6}$ Our data suggest that COVID-19 has not broken down these barriers, a number of which may be deeply entrenched within the specialty. ${ }^{26}$ Hospital teams were more likely to have cared for these patients and families. Only $23 \%$ of responding hospice inpatient and $30 \%$ of home palliative care teams had cared for dying patients with COVID-19 from ethnic minority groups. This is despite community and home care palliative care teams reporting that they have been being busier during the COVID-19 pandemic. $^{13}$ There are no comparable pre-COVID-19 data. The reasons for these inequalities, and how they relate to the distribution of ethnic minority groups in the population, and their palliative care needs, are unknown.

Our data demonstrate that visiting restrictions may have removed patients' psychosocial support and advocates, and their personal and professional translators for many, their only means of communication. The importance-among ethnic minority groups-of family visiting at the end of life has been highlighted previously. ${ }^{27} 28$ Also, the importance of interpreter use is recognised in providing high-quality clinical care for limited English-speaking patients. ${ }^{29}$ Verbal communication at the end of life is essential to meet basic care to assess a patient's clinical needs, and to consider important questions around prognosis and address fears. During times of illness and stress, competent English speakers may revert to their first language. Managing complex discussions about illness, prognosis and future expectations is difficult, but these difficulties appear to have been further compounded by personal protective equipment hindering the use of telephone interpreting services for patients from ethnic minority groups. Similarly, policies introduced, prohibiting physical contact with loved ones after death, may have disproportionately impacted ethnic minority groups who are more likely to conduct compulsory after-death rituals such as prayer in large groups with touching and washing of the body. Services repeatedly stressed the challenges in managing the distress related to these policies. While services were focused on delivering individualised care, the overarching changes in policy may have resulted in inequitable care. Gaps in routine data, national surveys and research may have contributed to neglect in UK policy with inadequate evidence on how to meet the health needs of those from ethnic minority groups.

While individualised care, the pillar on which palliative care is built, ideally should result in equitable delivery of care for those from ethnic minority groups, our findings demonstrate this was not in all cases. This is because there are other important factors which come into play. Structural racism is present if processes and organisational structures facilitate delivery of healthcare in a way that impacts one ethnic group more than another. ${ }^{26}$ Specifically, within the responses, we found patterns suggesting structural racism (eg, organisations' policies on restricted visiting despite the cultural and religious significance of this to some groups; policies that prevented compulsory after-death rituals; the disproportionate impact of visiting policies on communication for people from ethnic minority groups). Structural racism is present if organisations do not assess the impact of their policies and practices and mitigate or put safeguards in place when policies are found to adversely affect certain ethnic groups. ${ }^{30}$ Our data demonstrated no evidence of systematic assessment of the impact of these policies. Solutions to mitigate against increased distress experienced by those from ethnic minority groups were limited in both number and effectiveness.

For people from ethnic minority groups, the building blocks that should inform individualised care have been shown to be absent during clinical encounters that cannot be replayed. This includes the delivery of culturally congruent care. Our study suggests that there may have been instances where the palliative care response to COVID-19 may not have been universally culturally congruent. For example, there were instances where individuals completing the survey appeared to have preconceived cultural and religious reductionist views about certain ethnic groups. ${ }^{31}$ In addition, there was an expectation that the patient and family from the ethnic minority group needed to explain their needs and if communication did not go well, there appeared to be a deficit model directed at the patient and family. While participatory action from all parties (including patients and families) is required to deliver equitable healthcare, the duty should be on services to ensure that they are delivering culturally congruent care and to actively reach out to patients and families when they are highly vulnerable. In our survey, services reported that families were 'closing the doors' even when services reached out. What is not clear is whether this was occurring for the same reasons previously documented (lack of awareness, language barriers; cultural issues and culturally insensitive services) ${ }^{43}$ or whether this was specifically related to the pandemic (eg, fear of infection, mistrust of healthcare professionals/systems, fear of rationing). While previous literature has also noted inequitable care despite a focus on individualised care in other 
marginalised groups, ${ }^{33}$ our study is the first to note this in those from ethnic minority groups.

For care to be equitable, there need to be socially, culturally and religiously informed building blocks on which individualised care is built. Making sure these building blocks are in place will require a 'centring at the margins' approach - that is, a shift of viewpoint from the 'served' perspective to that of the 'underserved' group, in this case towards those from ethnic minority groups. There is an obligation to remove or minimise the disadvantage experienced by people due to their culture and ethnicity and take steps to meet the needs of these groups where these are different. As we move to a para-COVID-19 period, palliative care services need to urgently define, operationalise and accurately measure need in relation to use to draw conclusions about the presence or absence of inequities.

Importantly, all palliative care healthcare providers and services should consider how their attitudes, actions and delivery of care affect patients and families from ethnic minority groups and how they may disproportionately adversely impact and contribute to inequity of palliative care access, delivery and outcomes. In developing solutions, it is important that they are designed in concert with the population they are intended to serve. Moreover, they should be aimed at achieving long-term institutional culture change and avoid a deficit model where challenges and solutions to meet them are focused exclusively on the individual and their family. We examine the gap between equal and equitable care and provide recommendations in figure 1 . While we have focused on ethnic minority groups, our recommendations are relevant for all 'underserved' groups.

\section{Strengths}

This study is the first to examine across the four nations of the UK, how palliative care services are delivering care to patients and families from ethnic minority groups. Our data are the first to begin to understand the impact of 'one size fits all' policies on those from ethnic minority groups at the end of life and the first to examine the impact of urgent policies on marginalised and disproportionately adversely affected groups during the COVID-19 pandemic. Our survey was large (277 responses) with a high response rate $(76 \%$ of UK hospice services responding). The research team comprised an ethnically diverse group and a patient and carer from ethnic minority backgrounds were involved in critical reflection of the findings.

\section{Limitations}

Surveys were completed by clinical leads at the services/ hospices. Therefore, the data reflect their perceptions. We did not engage directly with patients or families from ethnic minority groups. There may have been ambiguity in the questions asked which led to different interpretations and therefore responses may not reflect actual clinical practice or views. Some of the responses lacked detail and there was a lack of responses from some areas with high proportions of ethnic minority groups (eg, the Midlands) and it was not clear what proportion of patients seen were from an ethnic minority group for those responding. The survey was conducted early in the pandemic and some services

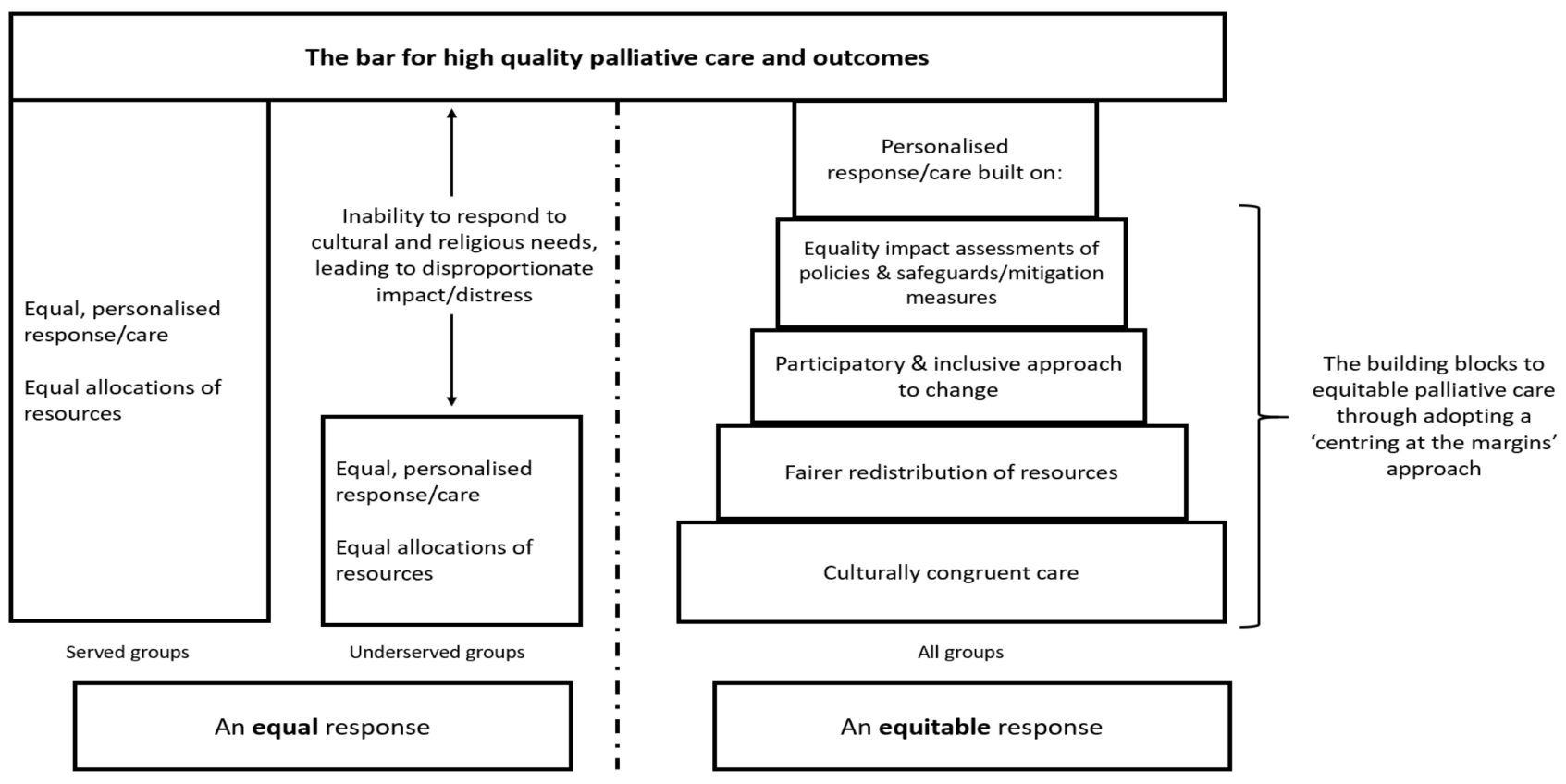

Figure 1 The delivery of equal palliative care during the COVID-19 pandemic and recommendations for equitable care for the future. 
may not have had many patients with COVID-19 at time of completion. Responses may have been subject to social desirability bias. We do not know whether services who did not respond had different experiences, with more or fewer challenges. While free text comments are a useful source of information, ${ }^{34}$ they may not represent all respondents.

\section{What's next/policy recommendations}

Within the UK, all public authorities and organisations have a legal duty to consider how their policies or decisions affect those from ethnic minority groups. ${ }^{35}$ An equality impact assessment is a legal requirement designed to help organisations ensure that their policies, practices and decision-making processes are fair and do not unfairly disadvantage protected groups. ${ }^{35}$ Where policies are found to unfairly disadvantage a protected group, safeguards and mitigation measures should be introduced. ${ }^{35}$ This may include flexibility in policies to take account of patients' communication and religious needs and the introduction of videoconferencing via tablets to facilitate communication for family, professional translation services and religious support. While policies introduced rapidly during the first wave of the COVID-19 pandemic may have been justified by the legitimate aim of protecting the general public, all services now need to urgently assess the impact of these and future policies on patients and families from ethnic minority groups. This should include specifically targeting issues around language and distress caused by 'one size fits all' policies. Formal safeguards and mitigation against the negative impact of these policies on these groups, beyond a sole focus on individualised care, are urgently needed. While our work is focused on palliative care services, our recommendations are relevant and timely for all healthcare specialties and settings. While these data are UK based, recommendations would potentially be applicable internationally.

\section{CONCLUSION}

Policies introduced during the COVID-19 pandemic may have adversely impacted those from certain ethnic minority groups, making these at-risk populations even more vulnerable. Furthermore, the traditional palliative care focused on individual care may be insufficient to provide equitable care. During the para-COVID period, systemic steps, including equality impact assessments, are urgently needed to identify, label and address inequities to ensure favourable experience and outcomes at the end of life for all patients and their families.

Twitter Sabrina Bajwah@SBajwah, Nancy Preston@ nancypreston16, Rachel Cripps@Rachelloucripps, Katherine E Sleeman@kesleeman and Catherine Walshe@cewalshe

Acknowledgements This study was part of CovPall, a multinational study supported by the Medical Research Council, National Institute for Health Research (NIHR)
Applied Research Collaboration South London and Cicely Saunders International. We thank all collaborators and advisors. We thank all participants, partners, PPI members and our Study Steering Group. We gratefully acknowledge technical assistance from the Precision Health Informatics Data Lab group (https://phidatalab.org) at NIHR Biomedical Research Centre at South London and Maudsley NHS Foundation Trust and King's College London for the use of REDCap for data capture.

Collaborators CovPall study team: Professor Irene J Higginson (Chief Investigator), Dr Sabrina Bajwah (Co-I), Dr Matthew Maddocks (Co-I), Professor Fliss Murtagh (Co-I), Professor Nancy Preston (Co-I), Professor Katherine E Sleeman (Co-I), Professor Catherine Walshe (Co-I), Professor Lorna K Fraser (Co-I), Dr Mevhibe B Hocaoglu (Co-I), Dr Adejoke Oluyase (Co-I), Dr Andrew Bradshaw, Lesley Dunleavy and Rachel L Cripps. CovPall study partners: Hospice UK, Marie Curie, Sue Ryder, Palliative Outcome Scale Team, European Association of Palliative Care (EAPC), Together for Short Lives and Scottish Partnership for Palliative Care.

Contributors $\mathrm{IJH}$ is the grant holder and chief investigator. KES, MM, FEMM, CW, NP, LKF, SB, MBH and AO are coapplicants for funding. IJH and $\mathrm{CW}$, with critical input from all authors, wrote the protocol for the CovPall study. $\mathrm{MBH}, \mathrm{AO}$ and $\mathrm{RC}$ coordinated data collection and liaised with centres, with input from $\mathrm{IJH}$. JK, JH, SB and $\mathrm{AO}$ analysed the data. All authors had access to all study data, discussed the interpretation of findings and take responsibility for data integrity and analysis. SB, FEMM, CW, AB, LKF, JK and JH drafted the manuscript. All authors provided critical revision of the manuscript for important intellectual content. SB and IJH are guarantors of the study.

Funding $\mathrm{IJH}$ is a National Institute for Health Research (NIHR) Emeritus Senior Investigator and is supported by the NIHR Applied Research Collaboration (ARC) South London (SL) at King's College Hospital National Health Service Foundation Trust. IJH leads the Palliative and End of Life Care theme of the NIHR ARC SL and coleads the national theme in this. MM is funded by an NIHR Career Development Fellowship (CDF-2017-10-009) and NIHR ARC SL. LEF is funded by an NIHR Career Development Fellowship (award CDF-2018-11-ST2-002). KES is funded by an NIHR Clinician Scientist Fellowship (CS-2015-15-005). RC is funded by Cicely Saunders International. FEMM is an NIHR Senior Investigator. $\mathrm{MBH}$ is supported by the NIHR ARC SL.

Disclaimer The views expressed in this article are those of the authors and not necessarily those of the NIHR or the Department of Health and Social Care.

Competing interests None declared.

Patient consent for publication Not required.

Ethics approval Ethical approval was obtained from King's College London Research Ethics Committee (LRS-19/2018541).

Provenance and peer review Not commissioned; externally peer reviewed.

Data availability statement Data are available upon reasonable request. Applications for use of the survey data can be made for up to 10 years, and will be considered on a case-by-case basis on receipt of a methodological sound proposal to achieve aims in line with the original protocol. The study protocol is available on request. All requests for data access should be addressed to the chief investigator via the details on the CovPall website (https://www.kcl.ac.uk/cicelysaunders/research/ evaluating/covpall-study and palliativecare@kcl.ac.uk) and will be reviewed by the Study Steering Group.

Open access This is an open access article distributed in accordance with the Creative Commons Attribution 4.0 Unported (CC BY 4.0) license, which permits others to copy, redistribute, remix, transform and build upon this work for any 
purpose, provided the original work is properly cited, a link to the licence is given, and indication of whether changes were made. See: https://creativecommons.org/licenses/by/4.0/.

\section{ORCID iDs}

Sabrina Bajwah http://orcid.org/0000-0001-5338-8107 Jonathan Koffman http://orcid.org/0000-0001-8513-5681 Andy Bradshaw http://orcid.org/0000-0003-1717-1546 Lorna K Fraser http://orcid.org/0000-0002-1360-4191 Adejoke Oluyase http://orcid.org/0000-0002-1506-7262 Katherine E Sleeman http://orcid.org/0000-0002-9777-4373 Irene J Higginson http://orcid.org/0000-0002-3687-1313 Catherine Walshe http://orcid.org/0000-0002-4531-8608 Fliss E M Murtagh http://orcid.org/0000-0003-1289-3726

\section{REFERENCES}

1 Public Health England. Disparities in the risk and outcomes of COVID-19; 2020.

2 Public Health England. Employment by occupation: Office of National Statistics, 2020. Available: https://www.ons.gov.uk/ employmentandlabourmarket/peopleinwork/employmentan demployeetypes/datasets/employmentbyoccupationemp04 [Accessed 03 Aug 2021].

3 Office of National Statistics. Coronavirus (COVID-19) related deaths by ethnic group, England and Wales: 2 March 2020 to 15 May 2020, 2020. Available: https://www.ons.gov.uk/ peoplepopulationandcommunity/birthsdeathsandmarriages/ deaths/articles/coronavirusrelateddeathsbyethnicgroupengland andwales/2march2020to10april2020 [Accessed 03 Aug 2021].

4 Koffman J, Burke G, Dias A, et al. Demographic factors and awareness of palliative care and related services. Palliat Med 2007;21:145-53.

5 Koffman J, Ho YK, Davies J, et al. Does ethnicity affect where people with cancer die? a population-based 10 year study. PLoS One 2014;9:e95052.

6 Tobin J, Rogers A, Winterburn I, et al. Hospice care access inequalities: a systematic review and narrative synthesis. BMJ Support Palliat Care 2021. doi:10.1136/ bmjspcare-2020-002719. [Epub ahead of print: 19 Feb 2021].

7 LoPresti MA, Dement F, Gold HT. End-Of-Life care for people with cancer from ethnic minority groups: a systematic review. Am J Hosp Palliat Care 2016;33:291-305.

8 Ornstein KA, Roth DL, Huang J, et al. Evaluation of racial disparities in hospice use and end-of-life treatment intensity in the REGARDS cohort. JAMA Netw Open 2020;3:e2014639.

9 GOV.UK. Writing about ethnicity, 2021. Available: https:// www.ethnicity-facts-figures.service.gov.uk/style-guide/writingabout-ethnicity

10 Chidiac C, Feuer D, Flatley M, et al. The need for early referral to palliative care especially for black, Asian and minority ethnic groups in a COVID-19 pandemic: findings from a service evaluation. Palliat Med 2020;34:1241-8.

11 Etkind SN, Bone AE, Gomes B, et al. How many people will need palliative care in 2040? Past trends, future projections and implications for services. BMC Med 2017;15:1-10.

12 Rees PWP, Clark S, Lomax NP. The future is diversity: New forecasts for the UK's ethnic groups. European Population Conference, Mainz, Germany, 2016.

13 Oluyase AO, Hocaoglu M, Cripps RL, et al. The challenges of caring for people dying from COVID-19: a multinational, observational study (CovPall). J Pain Symptom Manage 2021;56.

14 von Elm E, Altman DG, Egger M, et al. The strengthening the reporting of observational studies in epidemiology (STROBE) statement: guidelines for reporting observational studies. Int $J$ Surg 2014;12:1495-9.
15 Eysenbach G. Improving the quality of web surveys: the checklist for reporting results of Internet E-Surveys (cherries). $J$ Med Internet Res 2004;6:e132.

16 Patridge EF, Bardyn TP. Research electronic data capture (REDCap). J Med Libr Assoc 2018;106:142.

17 Garcia J, Evans J, Reshaw M. "Is There Anything Else You Would Like to Tell Us"- Methodological Issues in the Use of Free-Text Comments from Postal Surveys. Qual Quant 2004;38:113-25.

18 O'Cathain A, Thomas KJ. "Any other comments?" Open questions on questionnaires-a bane or a bonus to research? BMC Med Res Methodol 2004;4:1-7.

19 Braun V, Clarke V. One size fits all? what counts as quality practice in (reflexive) thematic analysis? Qual Res Psychol 2021;18:328-52.

20 Blaikie N. Confounding issues related to determining sample size in qualitative research. Int J Soc Res Methodol 2018;21:635-41.

21 Khunti K, Routen A, Pareek M. The language of ethnicity. BMJ $2020 ; 371$.

22 Hospice UK. Facts and figures. About hospice care, 2021. Available: https://www.hospiceuk.org/about-hospice-care/ media-centre/facts-and-figures

23 McCormack LA, McBride CM, Paasche-Orlow MK. Shifting away from a deficit model of health literacy. . Taylor \& Francis, 2016: 21. 4-5.

24 Culyer AJ. Equity - some theory and its policy implications. $J$ Med Ethics 2001;27:275-83.

25 World Health Organisation. A conceptual framework for action on the social determinants of health. Geneva, Switzerland WHO Document Production Services; 2010. https://www.who.int/social_determinants/corner/SDHDP2.pdf? ua $=1$ [Accessed 1 Sep 2021] .

26 Hussain JA, Koffman J, Bajwah S. Invited editorials. Palliat Med 2021;35:810-3.

27 Donnelly S, Prizeman G, Coimín Diarmuid Ó, et al. Voices that matter: end-of-life care in two acute hospitals from the perspective of bereaved relatives. BMC Palliat Care 2018;17:1-13.

28 Koffman J, Morgan M, Edmonds P, et al. Meanings of happiness among two ethnic groups living with advanced cancer in South London: a qualitative study. Psychooncology 2013;22:1096-103.

29 Ngo-Metzger Q, Sorkin DH, Phillips RS, et al. Providing high-quality care for limited English proficient patients: the importance of language concordance and interpreter use. J Gen Intern Med 2007;22 Suppl 2:324-30.

30 Bailey ZD, Feldman JM, Bassett MT. How Structural Racism Works - Racist Policies as a Root Cause of U.S. Racial Health Inequities. N Engl J Med 2021;384:768-73.

31 Koffman J. Servicing multi-cultural needs at the end of life. $J$ Ren Care 2014;40 Suppl 1:6-15.

32 Evans N, Meñaca A, Andrew EVW, et al. Systematic review of the primary research on minority ethnic groups and end-oflife care from the United Kingdom. J Pain Symptom Manage 2012;43:261-86.

33 Bristowe K, Marshall S, Harding R. The bereavement experiences of lesbian, gay, bisexual and/or trans* people who have lost a partner: a systematic review, thematic synthesis and modelling of the literature. Palliat Med 2016;30:730-44.

34 York GS, Churchman R, Woodard B, et al. Free-text comments: understanding the value in family member descriptions of hospice caregiver relationships. Am J Hosp Palliat Care 2012;29:98-105.

35 GOV.UK. Equality Act 2010: guidance, 2013. Available: https://www.gov.uk/guidance/equality-act-2010-guidance [Accessed 3 Aug 2021]. 